\title{
Disease-specific survival of patients with invasive cribriform and intraductal prostate cancer at diagnostic biopsy
}

\author{
Charlotte F Kweldam ${ }^{1}$, Intan P Kümmerlin ${ }^{1}$, Daan Nieboer ${ }^{2}$, Esther I Verhoef ${ }^{1}$, \\ Ewout W Steyerberg ${ }^{2}$, Theodorus H van der Kwast ${ }^{3}$, Monique J Roobol ${ }^{4}$ and \\ Geert J van Leenders ${ }^{1}$
}

${ }^{1}$ Department of Pathology, Erasmus Medical Centre, Rotterdam, The Netherlands; ${ }^{2}$ Department of Public Health, Erasmus Medical Centre, Rotterdam, The Netherlands; ${ }^{3}$ Laboratory Medicine Program, University Health Network, Toronto, ON, Canada and ${ }^{4}$ Department of Urology, Erasmus Medical Centre, Rotterdam, The Netherlands

\begin{abstract}
Invasive cribriform and intraductal carcinoma in radical prostatectomy specimens have been associated with an adverse clinical outcome. Our objective was to determine the prognostic value of invasive cribriform and intraductal carcinoma in pre-treatment biopsies on time to disease-specific death. We pathologically revised the diagnostic biopsies of 1031 patients from the first screening round of the European Randomized Study of Screening for Prostate Cancer (1993-2000). Ninety percent of all patients $(n=923)$ had received active treatment, whereas $10 \%(n=108)$ had been followed by watchful waiting. The median follow-up was 13 years. Patients who either had invasive cribriform growth pattern or intraductal carcinoma were categorized as CR/IDC+. The outcome was disease-specific survival. Relationships with outcome were analyzed using multivariable Cox regression and log-rank analysis. In total, 486 patients had Gleason score $6(47 \%)$ and 545 had $\geq 7$ (53\%). The 15-year disease-specific-survival probabilities were $99 \%$ in Gleason score $6(n=486), 94 \%$ in CR/IDC - Gleason score $\geq 7(n=356)$ and $67 \%$ in CR/IDC+ Gleason score $\geq 7(n=189)$. CR/IDC - Gleason score $3+4=7$ patients did not have statistically different survival probabilities from those with Gleason score $6(P=0.30)$, while CR/IDC+ Gleason score $3+4=7$ patients did $(P<0.001)$. In multivariable analysis, $C R / I D C+$ status was independently associated with a poorer disease-specific survival (HR 2.6, 95\% Cl 1.4-4.8, $P=0.002$ ). We conclude that CR/IDC+ status in prostate cancer biopsies is associated with a worse disease-specific survival. Our findings indicate that men with biopsy CR/IDC - Gleason score $3+4=7$ prostate cancer could be candidates for active surveillance, as these patients have similar survival probabilities to those with Gleason score 6.

Modern Pathology (2016) 29, 630-636; doi:10.1038/modpathol.2016.49; published online 4 March 2016
\end{abstract}

The management of newly diagnosed prostate cancer is challenging because of its heterogeneity in histology, genetics, and clinical outcome. Today, clinical decision-making mostly depends upon serum prostate-specific antigen (PSA) level, clinical tumor stage, and pathologic biopsy Gleason score-a grading system based on architectural tumor patterns. Although patients with the lowest Gleason scores $\leq 6$ have an excellent outcome, those with the highest Gleason scores (9-10) have the worst. ${ }^{1}$

Correspondence: Dr CF Kweldam, MD, Department of Pathology, Erasmus Medical Centre, Postbus 2040, 3000 CA Rotterdam, The Netherlands.

E-mail: c.kweldam@erasmusmc.nl

Received 1 December 2015; revised 28 January 2016; accepted 28 January 2016; published online 4 March 2016
The clinical outcome of Gleason score 7 prostate cancer patients is highly variable. Improving risk assessment is of particular interest, as Gleason score 7 prostate cancer on biopsy is an important clinical threshold for active treatment. Recent studies have suggested that the broad contemporary definition of the Gleason grade 4 pattern may be one of the explanations for the variable outcomes of patients with Gleason score 7 prostate cancer. ${ }^{2-5}$ Architecturally, four Gleason grade 4 growth patterns are recognized: ill-formed, fused, glomeruloid, and cribriform. ${ }^{1,6}$ Recently, cribriform pattern has been associated with adverse outcome after radical prostatectomy in Gleason score 7 prostate cancer. ${ }^{2-5}$

In recent years, the clinical relevance of intraductal carcinoma of the prostate-a high-risk lesion defined as malignant epithelium filling large acini or 
ducts with preservation of basal cells-has been acknowledged. Although not included in the Gleason score, intraductal carcinoma has been associated with high Gleason scores, advanced tumor stage, biochemical relapse, and distant metastasis. ${ }^{7-12}$ Intraductal carcinoma can, however, microscopically mimic invasive cribriform carcinoma requiring additional immunohistochemistry for their distinction. Studies on the prognostic value of invasive cribriform and intraductal carcinoma have mostly been based on radical prostatectomy specimens. ${ }^{2-5,11,13}$ The aim of this study was to determine the prognostic value of invasive cribriform and intraductal carcinoma in diagnostic biopsies on time to disease-specific death.

\section{Materials and methods}

\section{Patient Selection}

We included all 1078 men from the first screening round of the Dutch part of the European Randomized Study of Screening for Prostate Cancer (ERSPC), who had been diagnosed with prostate cancer between November 1993 and March 2000 in Erasmus Medical Centre, Rotterdam, The Netherlands. The trial protocol has been published previously. ${ }^{14,15}$ The ERSPC is an ongoing multicenter randomized screening trial that was initiated in the early 1990s to evaluate the effect of screening with PSA testing on disease-specific mortality rates. Exclusion criteria of the present study were unavailability of slides or paraffin blocks for review $(n=24)$ and presence of lymph node or distant metastasis at the time of diagnosis $(n=23)$, leaving 1031 patients for analysis.

\section{Pathological Evaluation}

Three investigators (CFK, IPK, and GJvL), who were blinded to patient information and outcome, revised all histopathological slides. For each biopsy core, we recorded tumor percentage, tumor length $(\mathrm{mm})$, Gleason score, presence of intraductal carcinoma, and presence of Gleason grade 4 and 5 growth patterns. ${ }^{1}$ The overall tumor percentage per patient was defined as the sum of total tumor length (mm) divided by the sum of total biopsy length ( $\mathrm{mm}$ ). The label CR/IDC+ was given to patients who either had invasive cribriform carcinoma, intraductal carcinoma or both, CR/IDC - to those who had neither. CR/IDC-specific tumor percentage per patient was defined as the sum of total length CR/IDC glands ( $\mathrm{mm}$ ) divided by the sum of total biopsy length (mm). Gleason grading was performed according to the 2014 ISUP recommendations. ${ }^{1}$ To distinguish invasive cribriform carcinoma from intraductal carcinoma and high-grade prostatic epithelial neoplasia (HGPIN) from intraductal carcinoma, we used morphological criteria as described by Guo et al. ${ }^{7}$ In case morphological distinction between invasive cribriform carcinoma and intraductal carcinoma was not certain $(105 / 193,54 \%)$, we applied high-molecular-weight-keratin immunohistochemistry to detect the presence of basal cells.

\section{Clinical Follow-up}

After diagnosis and initial treatment, patients were semi-annually monitored by chart review to assess potential progression and secondary treatments. The cause of death was evaluated by an independent cause-of-death committee, where deaths due to causes related to screening were also counted as prostate cancer deaths. ${ }^{16}$ Although data on the occurrence of distant metastases were available, we did not include this end point in our study, as these events largely overlapped with the number of disease-specific deaths.

\section{Statistical Analysis}

Continuous parameters were analyzed by the Mann-Whitney $U$-test or Kruskal-Wallis test, categorical parameters by the Pearson's chi-square $\left(\chi^{2}\right)$ test. Non-normally distributed continuous variables underwent log base 2 transformation such that effects related to a doubling in unit. We estimated survival probabilities using the Kaplan-Meier method. Unadjusted comparisons for survival time were made using log-rank tests with censoring of men lost to follow-up or dying of other causes. Crude and adjusted hazard ratios (HRs) for survival time were calculated using Cox proportional hazards regression. The concordance index (c-index) was used to quantify the ability of single variables and combinations of variables in multivariable models to discriminate between patients with and without the event of interest. ${ }^{17}$ The c-index takes values between 0.5 and 1 , where 0.5 indicates that the model is not better than chance classification and 1 means perfect discrimination. ${ }^{18}$ Regression models were compared using the likelihood-ratio test. All statistical analyses were performed in $\mathrm{R}$ version 3.1.2 ( $\mathrm{R}$, Vienna, Austria). Two-sided $P$-values of $<0.05$ were considered statistically significant.

\section{Results}

\section{Patient Characteristics}

The median age of the entire cohort $(N=1031)$ was 66 years (IQR 62-71) and the median followup was 13 years (IQR 9.4-16; Table 1). In total, 90\% of all patients $(n=923)$ had received active treatment, whereas $10 \%(n=108)$ had been followed by watchful waiting. A total of 496 patients died during follow-up, 72 of whom from prostate cancer. The majority $(53 \%)$ of patients had Gleason score $3+4=7$ or higher. Gleason score was positively associated with age, PSA level, tumor percentage, and 
Table 1 Patient and tumor characteristics $(N=1031)$

\begin{tabular}{|c|c|c|c|c|c|c|}
\hline & \multicolumn{5}{|c|}{ Mean (median, IQR) or $n(\%)$} & \multirow[b]{2}{*}{$\mathrm{P}$-value } \\
\hline & $\begin{array}{l}\text { Gleason score } 6 \\
\quad(\mathrm{n}=486)\end{array}$ & $\begin{array}{c}\text { Gleason score } \\
3+4=7(\mathrm{n}=310)\end{array}$ & $\begin{array}{l}\text { Gleason score } \\
4+3=7(\mathrm{n}=104)\end{array}$ & $\begin{array}{c}\text { Gleason score } 8 \\
(\mathrm{n}=64)\end{array}$ & $\begin{array}{l}\text { Gleason score } \\
9-10(\mathrm{n}=67)\end{array}$ & \\
\hline Age at diagnosis (years) & $66(66,61-70)$ & $66(67,62-71)$ & $68(69,65-71)$ & $68(69,66-72)$ & $67(67,64-71)$ & $<0.001^{\mathrm{a}}$ \\
\hline PSA level at diagnosis (ng/ml) & $5.8(4.7,3.5-6.9)$ & $8.8(5.8,4.0-9.0)$ & $15(8.6,4.7-18)$ & $19(11,6.2-17)$ & $16(9.4,5.4-16)$ & $<0.001^{\mathrm{a}}$ \\
\hline Percentage of positive cores (\%) & $31(29,17-43)$ & $2.9(3.0,2.0-4.0)$ & $50(43,29-71)$ & $55(50,40-71)$ & $62(57,43-86)$ & $<0.001^{\mathrm{a}}$ \\
\hline Tumor percentage $(\%)$ & $24(17,9.5-33)$ & $43(44,27-57)$ & $51(51,33-68)$ & $51(52,33-66)$ & $56(56,41-74)$ & $<0.001^{\mathrm{a}}$ \\
\hline \multicolumn{7}{|l|}{ Gleason grade 4 patterns } \\
\hline Ill-formed & & $227(73)$ & $63(85)$ & $51(80)$ & $64(96)$ & $<0.001^{\mathrm{b}}$ \\
\hline Fused & & $153(49)$ & $46(62)$ & $32(50)$ & $39(58)$ & $0.07^{\mathrm{b}}$ \\
\hline Cribriform & & $24(7.7)$ & $38(37)$ & $23(36)$ & $26(39)$ & $<0.001^{\mathrm{b}}$ \\
\hline Glomeruloid & & $33(11)$ & $14(19)$ & $13(20)$ & $11(16)$ & $0.02^{\mathrm{b}}$ \\
\hline \multicolumn{7}{|l|}{ Gleason grade 5 patterns } \\
\hline Single cells and strands & & & & $35(55)$ & $61(91)$ & $<0.001^{\mathrm{b}}$ \\
\hline Solid & & & & $3(4.7)$ & $16(24)$ & $0.002^{\mathrm{b}}$ \\
\hline Intraductal carcinoma & $4(0.82)$ & $41(13)$ & $44(42)$ & $18(28)$ & $32(48)$ & $<0.001^{\mathrm{b}}$ \\
\hline CR/IDC+ status & $4(0.82)$ & $54(17)$ & $60(58)$ & $33(52)$ & $42(63)$ & $<0.001^{\mathrm{b}}$ \\
\hline \multicolumn{7}{|l|}{ Primary treatment } \\
\hline Radical prostatectomy & $216(44)$ & $129(42)$ & $33(32)$ & $14(22)$ & $14(21)$ & $<0.001^{\mathrm{b}}$ \\
\hline Radiotherapy & $188(39)$ & $154(59)$ & $66(63)$ & $48(75)$ & $52(78)$ & $<0.001^{\mathrm{b}}$ \\
\hline Endocrine treatment & $2(0.41)$ & $3(0.97)$ & $1(0.96)$ & $1(1.6)$ & & \\
\hline Watchful waiting & $80(17)$ & $23(7.4)$ & $3(2.8)$ & $1(1.6)$ & $1(1.5)$ & $<0.001^{\mathrm{b}}$ \\
\hline $\begin{array}{l}\text { Radiotherapy and endocrine } \\
\text { treatment }\end{array}$ & & & $1(0.96)$ & & & \\
\hline Unknown & & $1(0.27)$ & & & & \\
\hline Prostate-cancer-specific deaths & $8(1.6)$ & $14(4.5)$ & $17(16)$ & $14(22)$ & $19(28)$ & \\
\hline
\end{tabular}

${ }^{a}$ Kruskal-Wallis test. ${ }^{b}$ Pearson's chi-square $\left(\chi^{2}\right)$ test.

percentage of positive cores. The most frequently observed Gleason grade 4 pattern in Gleason score $3+4=7$ or higher was ill-formed $(80 \%)$, followed by fused $(53 \%)$, cribriform $(20 \%)$, and glomeruloid $(15 \%)$. Presence of cribriform growth was the most discriminative Gleason grade 4 pattern between Gleason score $3+4=7$ and $4+3=7$ (7.7 vs $37 \%$, $\left.\chi^{2} \quad P<0.001\right)$. We found a similar association for intraductal carcinoma (13 vs $42 \%, \chi^{2} P<0.001$ ). Intraductal carcinoma co-existed with invasive cribriform carcinoma in 57 of 111 patients (51\%). Invasive cribriform and intraductal carcinoma were predominantly seen in Gleason score $4+3=7$ and higher prostate cancer. In total, 193 patients had CR/IDC+ status; the distribution among Gleason score is shown in Table 1. Most low-risk patients had undergone radical prostatectomy whereas high-risk patients had received radiotherapy.

\section{Prognostic Value of CR/IDC Status}

Presence of intraductal carcinoma (crude HR 7.6, 95\% CI $4.8-12 ; \quad P<0.001, \quad$ c-index $=0.697)$ and invasive cribriform carcinoma (crude HR 6.3, 95\% CI 3.9-10; $P<0.001$, c-index $=0.639$ ) were both significantly associated with worse disease-specific survival in univariate analyses. The combined CR/IDC+ status was also strongly associated with worse disease-specific survival (crude HR 11, 95\%
CI $6.6-18 ; \quad P<0.001, \quad$ c-index $=0.758$ ) and was similar if intraductal carcinoma and invasive cribriform carcinoma were analyzed as separate predictors in a model (c-index $=0.761$ ). When separating each Gleason score group for CR/IDC status the diseasespecific-survival probabilities were significantly lower in CR/IDC+ patients with Gleason score 3 $+4=7,8$, and 9-10 (Figure 1). Although we saw some evidence of lower survival probabilities in CR/IDC+ Gleason score $4+3=7$, differences between groups did not meet conventional levels of statistical significance (log rank $P=0.054$ ). The Gleason score 6 group contained only four CR/IDC+ patients (all intraductal carcinoma). CR/IDC - Gleason score $3+4=7$ patients did not have significantly different survival probabilities from those with Gleason score 6 (log rank $P=0.30$ ), while CR/IDC+ Gleason score 3 $+4=7$ patients had significantly worse survival probabilities than those with Gleason score 6 (log rank $P<0.001$ ) and CR/IDC - Gleason score $3+4=7$ (log rank $P=0.001$ ). The survival probabilities of CR/ IDC - patients with $4+3=7$ or higher were significantly lower than of those with Gleason score 6 (log rank $P<0.001, P=0.03$, and $P<0.001$, respectively). CR/IDC - Gleason score $4+3=7$ patients also had worse survival probabilities than those with CR/ IDC $-3+4=7(P=0.03)$. Although patients with CR/ IDC - Gleason score 9-10 had poorer survival probabilities than those with CR/IDC - Gleason score $3+4=7$ prostate cancer (log rank $P=0.001$ ), 
a

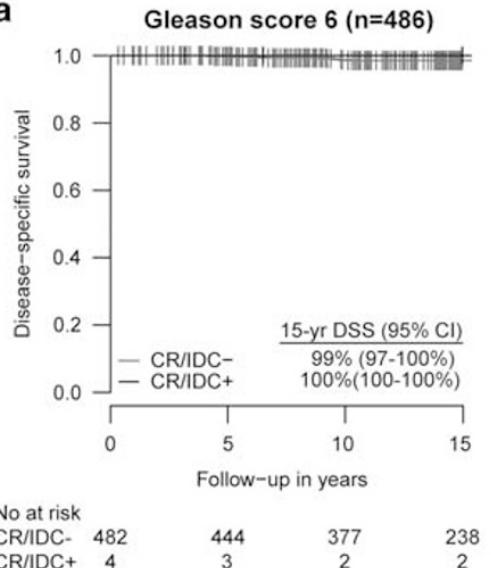

d

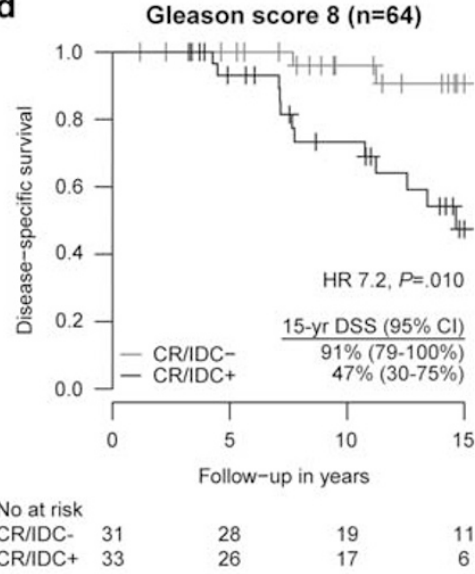

b

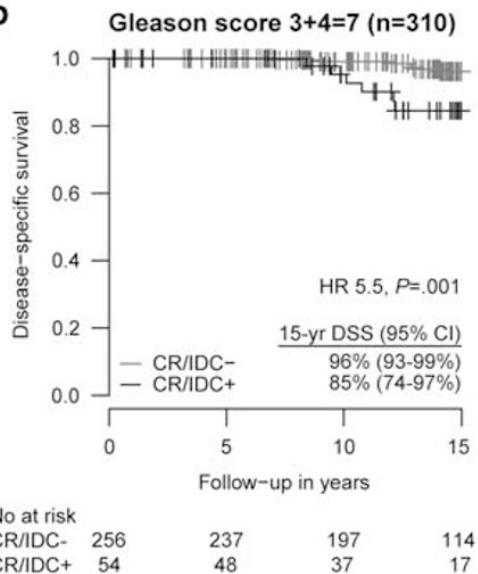

e

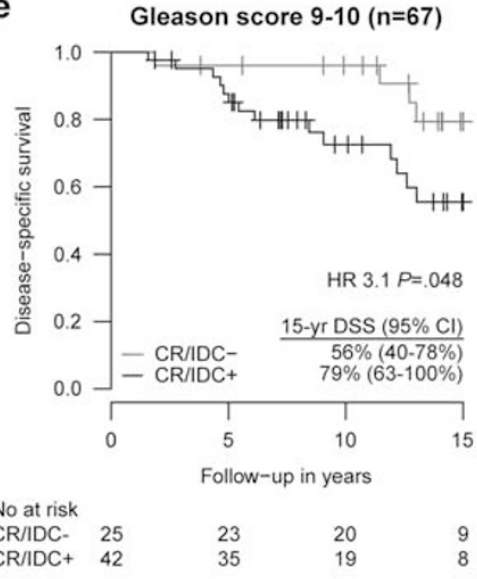

C

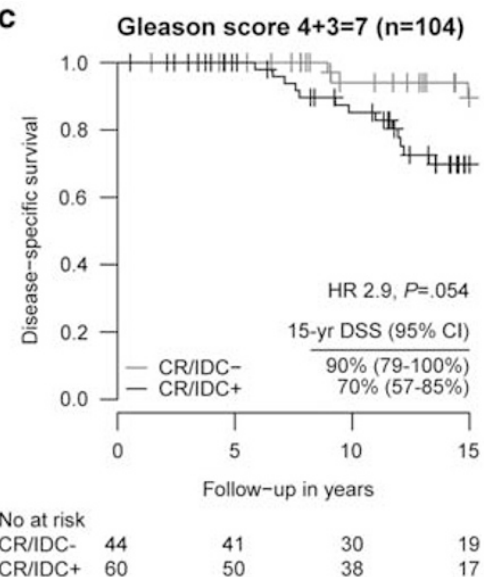

f

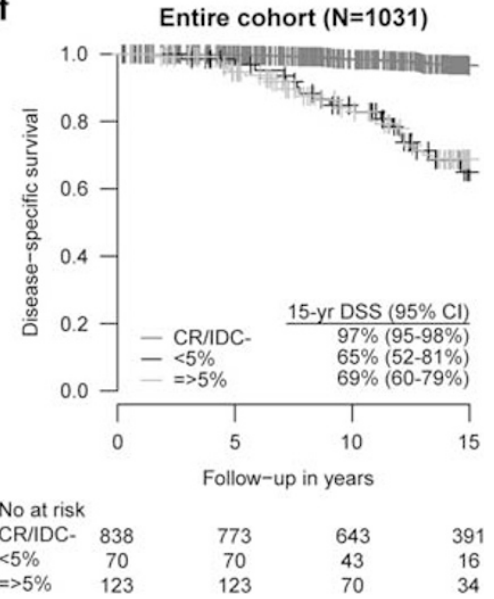

Figure 1 Kaplan-Meier disease-specific survival (DSS) according to Gleason score and CR/IDC status. (a) Gleason score 6. (b) Gleason score 3+4=7. (c) Gleason score 4+3=7. (d) Gleason score 8. (e) Gleason score 9-10. (f) DSS probabilities according to percentage of CR/IDC glands.

there was no statistical difference in disease-specificsurvival probabilities comparing CR/IDC - Gleason score 9-10 with CR/IDC - Gleason score $4+3=7$ and $\mathrm{CR} / \mathrm{IDC}$ - Gleason score 8 patients (log rank $P=0.41$ and $P=0.40$, respectively). In general, the 15-year disease-specific-survival probabilities were $94 \%$ (95\% CI 91-97\%) in CR/IDC - Gleason score $3+4=7$ or higher $(n=356)$ and $67 \%(95 \%$ CI $59-76 \%$ ) in CR/IDC+ Gleason score $3+4=7$ or higher $(n=189)$. The presence of CR/IDC growth affected disease-specific survival regardless of its extent (Figure 1f).

In a multivariable model, we analyzed the added prognostic value of CR/IDC status in combination with currently used clinically relevant variables, ie, age, PSA level, treatment modalities, Gleason score, tumor percentage, and percentage of positive cores. In the model without CR/IDC status, the following variables were independently associated with a worse disease-specific survival: PSA level, tumor percentage, percentage of positive cores, and Gleason score $4+3=7$ or higher (Table 2). After adding CR/IDC status into the model, Gleason score $4+3=7$ and 8 were not independently associated with a poorer disease-specific survival anymore. We found that the c-index significantly increased from 0.868 to 0.877 after CR/IDC status was added to the model (likelihood-ratio test, $P=0.001$ ). There was no statistically significant interaction between CR/IDC status and treatment (likelihood-ratio test, $P=0.14$ ) or CR/IDC status and Gleason score (likelihood-ratio test, $P=0.71$ ).

\section{Discussion}

The current study showed that CR/IDC status in diagnostic biopsies is associated with a worse disease-specific survival. Adding CR/IDC status to a predictive model resulted in a significantly better discriminative ability. The most interesting finding of our study was the overall good outcome of patients whose biopsies lacked CR/IDC growth, particularly in those with CR/IDC - Gleason score $3+4=7$, whose survival did not differ from patients with Gleason score 6 prostate cancer. We additionally found that presence of a limited CR/IDC tumor component $(\leq 5 \%)$ in biopsies was already associated with an unfavorable outcome. This finding is in line with the study of Trudel et $a l,{ }^{13}$ who showed that 
Table 2 Adjusted HRs on time to disease-specific death in a clinical setting: the added value of CR/IDC status $(N=1031)$

\begin{tabular}{|c|c|c|c|c|c|c|}
\hline & \multicolumn{3}{|c|}{ Model without CR/IDC status } & \multicolumn{3}{|c|}{ Model with CR/IDC status } \\
\hline & Adjusted HR & $95 \% C I$ & P-value & Adjusted HR & $95 \% C I$ & $\mathrm{P}$-value \\
\hline Age (years) & 0.99 & $0.94-1.0$ & 0.60 & 0.99 & $0.94-1.0$ & 0.63 \\
\hline PSA level $\left(\log _{2}\right)$ & $1.2^{\mathrm{a}}$ & $1.0-1.5$ & 0.02 & $1.2^{\mathrm{a}}$ & $1.0-1.5$ & 0.04 \\
\hline Percentage of positive cores $\left(\log _{2}\right)$ & $1.8^{\mathrm{a}}$ & $1.2-2.6$ & 0.006 & $1.6^{\mathrm{a}}$ & $1.0-2.4$ & 0.03 \\
\hline Tumor percentage $\left(\log _{2}\right)$ & $1.5^{\mathrm{a}}$ & $1.1-2.1$ & 0.02 & $1.4^{\mathrm{a}}$ & $1.0-2.0$ & 0.05 \\
\hline \multicolumn{7}{|l|}{ Gleason score } \\
\hline 6 & & Reference & & & Reference & \\
\hline $3+4=7$ & 1.2 & $0.48-3.1$ & 0.69 & 0.99 & $0.38-2.6$ & 0.99 \\
\hline $4+3=7$ & 3.1 & $1.2-8.0$ & 0.02 & 1.9 & $0.67-5.4$ & 0.23 \\
\hline 8 & 3.7 & $1.4-10$ & 0.01 & 2.3 & $0.78-6.9$ & 0.13 \\
\hline $9-10$ & 5.1 & $2.0-13$ & $<0.001$ & 3.3 & $1.2-9.3$ & 0.02 \\
\hline CR/IDC+ status & & & & 2.6 & $1.4-4.8$ & 0.002 \\
\hline Radical prostatectomy & 0.23 & $0.058-0.92$ & 0.04 & 0.26 & $0.064-1.0$ & 0.05 \\
\hline Radiotherapy & 1.3 & $0.40-4.5$ & 0.63 & 1.4 & $0.42-4.7$ & 0.58 \\
\hline
\end{tabular}

${ }^{\mathrm{a} P e r}$ doubling unit.

any amount of large cribriform or intraductal carcinoma was associated with shorter time to biochemical recurrence after radical prostatectomy.

In recent radical prostatectomy studies, intraductal carcinoma and invasive cribriform carcinoma have both been identified as independent prognostic factors. $^{2-5,11,13}$ To date, only few studies have analyzed the prognostic value of intraductal carcinoma in pre-treatment diagnostic biopsies. ${ }^{7-9}$ They showed that intraductal carcinoma is associated with high-grade and non-organ-confined prostate cancer in subsequent radical prostatectomies. ${ }^{7,8}$ In addition, Van der Kwast et $a l^{9}$ demonstrated that intraductal carcinoma was associated with shorter time to biochemical recurrence and distant metastasis after radiotherapy in intermediate- to high-risk prostate cancer patients.

Although CR/IDC status in our predictive model led to significantly better discriminative ability, the absolute c-indices in the models with and without CR/IDC status only differed marginally. CR/IDC status might not affect clinical decision-making in patients with Gleason score 8-10 since these patients will undergo active treatment either way. CR/IDC status could, however, be useful to stratify Gleason score $3+4=7$ patients for active surveillance or treatment. A drawback of the current Gleason grading system is its considerable inter-observer variability, in particular when distinguishing Gleason score $3+4=7$ from Gleason score 6 prostate cancer. ${ }^{19,20}$ Variability in assignment of grade is significantly related to the presence of ill-formed and fused growth patterns; these represented the majority of Gleason score $3+4=7$ prostate cancers in this study. Egevad et $a l^{21}$ found that cribriform growth was not statistically associated with Gleason score inter-observer variability among 337 pathologists. This indicates that CR/IDC status may be a more robust parameter for patient stratification than grading as either Gleason score 6 or $3+4=7$.
Although invasive cribriform carcinoma and intraductal carcinoma are two different pathologic entities, they may be related on a pathological and biological level..$^{22,23}$ Their morphologic distinction is often difficult requiring immunohistochemical staining for basal cells. Although presence of basal cells is strongly supportive of intraductal carcinoma, lack of basal cells is not pathognomonic for invasive cribriform growth; basal cells can be scattered and not be sampled in the tissue section, which is also known to occur in HGPIN. ${ }^{24}$ The use of combined CR/IDC status is practical for pathologic diagnosis since it does not affect prognostic value of separate entities nor requires additional immunohistochemistry. This is also in line with the latest 2014 ISUP recommendations on Gleason grading, in which Epstein et $a l^{1}$ advised that immunohistochemistry to distinguish invasive cribriform from intraductal carcinoma should only be considered in cases where the results of the studies would change the overall grade of the case, for example, in cases lacking other Gleason grade 4 patterns.

Several studies have reported on genetic abnormalities related to CR/IDC growth. Qian et al found gain of chromosome 7,12 , and Y, loss of chromosome 8, and extra copies of $c-M Y C$ in both cribriform HGPIN and invasive cribriform carcinoma, suggesting that these growth patterns are genetically more alike to Gleason grade 5 than Gleason grade 3 or 4 prostate cancer. ${ }^{25,26}$ Dawkins et $a l^{27}$ reported frequent losses of 8p22 and 16q23.1 in intraductal carcinoma. Bettendorf et $a l^{28}$ found that intraductal carcinoma has more frequent loss of TP53, RB1, and PTEN. Using break-point regions to infer phylogenetic relationships, Lindberg et $a l^{29}$ showed that the clone closely related to the metastases was found in intraductal carcinoma. We hypothesize that both invasive cribriform and intraductal carcinoma are architectural substrates of genetic aberrations associated with aggressive disease behavior. The fact 
that small CR/IDC components were already associated with worse outcome, could be explained by the emergence of aggressive tumor clones irrespective of their volume.

A limitation of the current study is the fact that the original ERSPC biopsy protocol included sextant biopsies, while current biopsy schemes are more extensive and increasingly MRI targeted reducing the chance of sampling artifact. Future research is needed to confirm that the prognostic value of CR/IDC status is similar in contemporary biopsy protocols. Another limitation is the difference in treatment modalities nowadays as compared to the 1990s. Low-risk patients in this study had mostly received active treatment, while active surveillance would have been an acceptable strategy nowadays. The strengths of the present study are its large number of patients with long-term follow-up, the use of disease-specific survival as an outcome measure, and the meticulous pathologic review. In conclusion, $\mathrm{CR} / \mathrm{IDC}+$ status in prostate cancer biopsies is independently associated with poorer diseasespecific survival. Our findings indicate that men with biopsy CR/IDC - Gleason score $3+4=7$ prostate cancer could be candidates for active surveillance, as these patients have similar survival probabilities to those with Gleason score 6.

\section{Disclosure/conflict of interest}

MJR has had travel, accommodations, or other expenses paid or reimbursed by Novartis, currently or during the past 2 years.

\section{References}

1 Epstein JI, Egevad L, Amin MB et al. The 2014 International Society of Urological Pathology (ISUP) Consensus Conference on Gleason Grading of Prostatic Carcinoma: Definition of Grading Patterns and Proposal for a New Grading System. Am J Surg Pathol 2016;40: 244-252.

2 Iczkowski KA, Torkko KC, Kotnis GR et al. Digital quantification of five high-grade prostate cancer patterns, including the cribriform pattern, and their association with adverse outcome. Am J Clin Pathol 2011;136:98-107.

3 Kryvenko ON, Gupta NS, Virani N et al. Gleason score 7 adenocarcinoma of the prostate with lymph node metastases: analysis of 184 radical prostatectomy specimens. Arch Pathol Lab Med 2013;137:610-617.

4 Dong F, Yang P, Wang C et al. Architectural heterogeneity and cribriform pattern predict adverse clinical outcome for Gleason grade 4 prostatic adenocarcinoma. Am J Surg Pathol 2013;37:1855-1861.

5 Kweldam CF, Wildhagen MF, Steyerberg EW et al. Cribriform growth is highly predictive for postoperative metastasis and disease-specific death in Gleason score 7 prostate cancer. Mod Pathol 2015;28:457-464.

6 Epstein JI, AW C Jr, Amin MB et al. ISUP Grading Committee The 2005 International Society of Urological Pathology (ISUP) Consensus Conference on Gleason
Grading of Prostatic Carcinoma. Am J Surg Pathol 2005;29:1228-1242.

7 Guo CC, Epstein JI. Intraductal carcinoma of the prostate on needle biopsy: histologic features and clinical significance. Mod Pathol 2006;19: 1528-1535.

8 Robinson BD, Epstein JI. Intraductal carcinoma of the prostate without invasive carcinoma on needle biopsy: emphasis on radical prostatectomy findings. J Urol 2010;184:1328-1333.

9 Van der Kwast T, Al Daoud N, Collette L et al. Biopsy diagnosis of intraductal carcinoma is prognostic in intermediate and high risk prostate cancer patients treated by radiotherapy. Eur J Cancer 2012;48: 1318-1325.

10 Watts K, Li J, Magi-Galluzzi C et al. Incidence and clinicopathological characteristics of intraductal carcinoma detected in prostate biopsies: a prospective cohort study. Histopathology 2013;63:574-579.

11 Kimura K, Tsuzuki T, Kato M et al. Prognostic value of intraductal carcinoma of the prostate in radical prostatectomy specimens. Prostate 2014;74:680-687.

12 Chen ZB, Chen N, Shen PF et al. The presence and clinical implication of intraductal carcinoma of prostate in metastatic castration resistant prostate cancer. Prostate 2015;75:1247-1254.

13 Trudel D, Downes MR, Sykes J et al. Prognostic impact of intraductal carcinoma and large cribriform carcinoma architecture after prostatectomy in a contemporary cohort. Eur J Cancer 2014;50: 1610-1616.

14 Roobol MJ, Schroder FH. European randomized study of screening for prostate cancer: achievements and presentation. BJU Int 2003;92 Suppl 2:117-122.

15 Schroder FH, Hugosson J, Roobol MJ et al. Screening and prostate-cancer mortality in a randomized European study. N Engl J Med 2009;360:1320-1328.

16 De Koning HJ, Blom J, Merkelbach JW et al. Determining the cause of death in randomized screening trial(s) for prostate cancer. BJU Int 2003;92 Suppl 2:71-78.

17 Harrell FE. Regression Modeling Strategies: with Applications to Linear Models, Logistic Regression, and Survival Analysis. Springer: New York, 2001, pp 256-258.

18 Steyerberg EW. Clinical prediction models: a practical approach to development, validation, and updating. In: Statistics for Biology and Health. Springer: New York, 2009, pp 260-270.

19 McKenney JK, Simko J, Bonham M et al. The potential impact of reproducibility of Gleason grading in men with early stage prostate cancer managed by active surveillance: a multi-institutional study. J Urol 2011;186:465-469.

20 Zhou M, Li JB, Cheng L et al. Diagnosis of "poorly formed glands" Gleason pattern 4 prostatic adenocarcinoma on needle biopsy: an interobserver reproducibility study among urologic pathologists with recommendations. Am J Surg Pathol 2015;39: 1331-1339.

21 Egevad L, Algaba F, Berney DM et al. Interactive digital slides with heat maps: a novel method to improve the reproducibility of Gleason grading. Virchows Arch 2011;459:175-182.

22 Cohen RJ, Wheeler TM, Bonkhoff $\mathrm{H}$ et al. A proposal on the identification, histologic reporting, and implications of intraductal prostatic carcinoma. Arch Pathol Lab Med 2007;131:1103-1109. 
23 Haffner MC, Weier C, Xu MM et al. Molecular evidence that invasive adenocarcinoma can mimic prostatic intraepithelial neoplasia (PIN) and intraductal carcinoma through retrograde glandular colonization. J Pathol 2016;238:31-41.

24 Shah IA, Schlageter MO, Stinnett P et al. Cytokeratin immunohistochemistry as a diagnostic tool for distinguishing malignant from benign epithelial lesions of the prostate. Mod Pathol 1991;4:220-224.

25 Qian J, Jenkins RB, Bostwick DG. Detection of chromosomal anomalies and c-myc gene amplification in the cribriform pattern of prostatic intraepithelial neoplasia and carcinoma by fluorescence in situ hybridization. Mod Pathol 1997;10:1113-1119.

26 Jenkins RB, Qian J, Lieber MM et al. Detection of c-myc oncogene amplification and chromosomal anomalies in metastatic prostatic carcinoma by fluorescence in situ hybridization. Cancer Res 1997;57:524-531.

27 Dawkins HJ, Sellner LN, Turbett GR et al. Distinction between intraductal carcinoma of the prostate (IDC-P), high-grade dysplasia (PIN), and invasive prostatic adenocarcinoma, using molecular markers of cancer progression. Prostate 2000;44:265-270.

28 Bettendorf O, Schmidt H, Staebler A et al. Chromosomal imbalances, loss of heterozygosity, and immunohistochemical expression of TP53, RB1, and PTEN in intraductal cancer, intraepithelial neoplasia, and invasive adenocarcinoma of the prostate. Genes Chromosomes Cancer 2008;47:565-572.

29 Lindberg J, Kristiansen A, Wiklund P et al. Tracking the origin of metastatic prostate cancer. Eur Urol 2015;67: 819-822. 